\title{
Quantization of Hamiltonian systems with a position dependent mass: Killing vector fields and Noether momenta approach
}

\author{
José F. Cariñena ${ }^{a)}$, Manuel F. Rañada $\dagger^{b)}$, and Mariano Santander $\ddagger^{c)}$ \\ $\dagger$ Departamento de Física Teórica and IUMA, Facultad de Ciencias \\ Universidad de Zaragoza, 50009 Zaragoza, Spain \\ $\ddagger$ Departamento de Física Teórica and IMUVa, Facultad de Ciencias \\ Universidad de Valladolid, 47011 Valladolid, Spain
}

October 6, 2017

\begin{abstract}
The quantization of systems with a position dependent mass (PDM) is studied. We present a method that starts with the study of the existence of Killing vector fields for the PDM geodesic motion (Lagrangian with a PDM kinetic term but without any potential) and the construction of the associated Noether momenta. Then the method considers, as the appropriate Hilbert space, the space of functions that are square integrable with respect to a measure related with the PDM and, after that, it establishes the quantization, not of the canonical momenta $p$, but of the Noether momenta $P$ instead. The quantum Hamiltonian, that depends on the Noether momenta, is obtained as an Hermitian operator defined on the PDM Hilbert space. In the second part several systems with position-dependent mass, most of them related with nonlinear oscillators, are quantized by making use of the method proposed in the first part.
\end{abstract}

Keywords: Position-dependent mass. Quantization. Killing vector fields. Noether Momenta Running title: Quantization, PDM, Killing vector fields and Noether Momenta.

AMS classification: 70S05;81Q80 ; 81U15

PACS numbers: 03.65.-w

${ }^{a)}$ E-mail address: jfc@unizar.es

b) E-mail address: mfran@unizar.es

${ }^{c)}$ E-mail address: mariano.santander@uva.es 


\section{Introduction}

Suppose we are given a one-dimensional system described, in terms of a coordinate $x$, by a Lagrangian

$$
L=\frac{1}{2} m(x) \dot{x}^{2}-V(x), \quad x \in \mathbb{R}, \quad m(x)>0,
$$

that is, the usual constant mass $m$ is replaced by a strictly positive function of the position; then the Hamiltonian $H$, that is given by

$$
H(x, p)=\frac{1}{2} \frac{1}{m(x)} p^{2}+V(x),
$$

is correctly defined. The point is that there is an important problem with the construction of the quantum version of $H$, that is, with the transition $H \rightarrow \widehat{H}$ from the classical system to the quantum one, because if the mass $m$ becomes a function of the spatial coordinate, $m=m(x)$, then the quantum version of the mass no longer commutes with the momentum. Therefore, different forms of presenting the kinetic term in the Hamiltonian $H$, as for example

$$
T=\frac{1}{4}\left[\frac{1}{m(x)} p^{2}+p^{2} \frac{1}{m(x)}\right], \quad T=\frac{1}{2}\left[\frac{1}{\sqrt{m(x)}} p^{2} \frac{1}{\sqrt{m(x)}}\right], \quad T=\frac{1}{2}\left[p \frac{1}{m(x)} p\right],
$$

are equivalent at the classical level but they lead to different and nonequivalent Schrödinger equations.

This problem is important mainly for two reasons.

(i) There are a certain number of important areas, mainly related with problems on condensed-matter physics (electronic properties of semiconductors, liquid crystals, quantum dots, etc), in which the behaviour of the system depends of an effective mass that is position-dependent.

(ii) From a more conceptual viewpoint, the ordering of factors in the transition from a commutative to a noncommutative formalism is an old question that remains as an important open problem in the theory of quantization.

This question has been studied by many authors from different points of view that, in most of cases, make use of the formalism $(\alpha, \beta, \gamma)$ that we present in the next paragraphs.

\section{$1.1 \quad$ Formalism $(\alpha, \beta, \gamma)$}

The formalism we call $(\alpha, \beta, \gamma)$ makes use as a starting point of a rather general form of the kinetic term that includes several possible alternatives. The main idea is to represent $T$ as the following expression depending on three (related) parameters

$$
T_{\alpha \beta \gamma}=\frac{1}{4}\left(m^{\alpha} p m^{\beta} p m^{\gamma}+m^{\gamma} p m^{\beta} p m^{\alpha}\right), \quad \alpha+\beta+\gamma=-1 .
$$

It was introduced by von Roos in [1] (generalizing a previous study by BenDaniel et al [2]) and then used by other different authors [3]-24]. We quote the following text appearing in [9]: 
One of the well-known problems of the position-dependent effective mass (PDEM) Schrödinger equation (SE) consists on the momentum and mass-operator noncommutativity and the resultant ordering ambiguity in the kinetic energy term. To cope with this difficulty, it is advantageous to use the von Roos general two-parameter form of the effective mass kinetic energy operator [1] which has an inbuilt Hermiticity and contains other plausible forms as special cases.

Even if not explicitly stated, it is assumed that the configuration space is $\mathbb{R}$ with its natural coordinate $x$, the Hilbert space of the quantum system is $L^{2}(\mathbb{R}, d x)$ and that $p$ is represented by the differential operator $p=-i \hbar d / d x$ in such space, and this is the reason for the symmetrization in the expression of $T_{\alpha \beta \gamma}$.

It is important to remark that different choices of the parameters, $\alpha, \beta$, and $\gamma$, (known as von Roos ambiguity parameters) lead to distinct non-equivalent quantum Hamiltonians. Therefore, this formalism admits many particular cases; we first mention that BenDaniel et al 2 proposed (in a study previous to that of von Roos) ( $\alpha=0, \beta=-1, \gamma=0)$; other choices are for example, Zhu et al 3 which use the choice $(\alpha=-1 / 2, \beta=0, \gamma=-1 / 2)$, Li et al 4] $(\alpha=0, \beta=\gamma=-1 / 2)$, and Mustafa et al [18] $(\alpha=-1 / 4, \beta=-1 / 2, \gamma=-1 / 4)$. We also note that some authors simplify the number of parameters and make use of a simpler expression: For example in Ref. [14, [17, the expression of $T$ is

$$
T_{a b}=\frac{1}{2}\left(m^{a} p m^{2 b} p m^{a}\right), \quad a+b=-1 / 2,
$$

while in Ref. [19] the expression of $T$ uses only a parameter $r=a$ and then $2 b=-1-2 r$.

Lévy-Leblond studied this problem in [25] and, after analyzing some different possible quantizations, he proposed (by making use of some arguments related with the Galilei transformations) as the most appropriate form for $T_{\alpha \beta \gamma}$ to carry out the quantization the choice $(\alpha=0, \beta=-1, \gamma=0)$ that coincides with the one used in [2]. That is,

$$
T_{\mathrm{LL}}=\frac{1}{2}\left(p \frac{1}{m(x)} p\right)
$$

He then asserts that if another different form of $T_{\alpha \beta \gamma}$ is chosen, then it is convenient to introduce an effective potential $V_{\text {eff }}(x)$ that can be obtained by addition to the potential $V(x)$ of an additional term $U(x)$ depending on $m(x)$ and of its derivatives $m^{\prime}(x)$ and $m^{\prime \prime}(x)$ with $(\alpha, \beta, \gamma)$-dependent coefficients (in fact, this is a translation of the problem from the kinetic term $T$ into the potential $V$ ). As an example, if the kinetic term $T$ is written as

$$
T=\frac{1}{4}\left(\frac{1}{m(x)} p^{2}+p^{2} \frac{1}{m(x)}\right)
$$

then the Hamiltonian must be modified by replacing $V(x)$ with the following effective potential

$$
V_{\mathrm{eff}}=V(x)-\frac{1}{2} \frac{m^{\prime 2}}{m^{3}}+\frac{1}{4} \frac{m^{\prime \prime}}{m^{2}} .
$$

A certain number of authors have studied this question and shown a certain preference for Lévy-Leblond choice [26] 38] (with or without the effective potential). 


\section{$1.2 \quad$ Purpose and structure of the paper}

The aim of this paper is to present a method of quantization of Hamiltonian systems with PDM that, although it is not totally new (in fact, it has been already applied in some very particular cases [39, 40]), it is now presented in a general form. It is formulated starting with two important points. First, we consider that if the constant mass $m$ is replaced by a positive function then it is convenient to introduce this function as a factor on the metric and this property has an important influence in the form of the Hilbert space of wave functions, and second, we consider that for obtaining the quantum Hamiltonian a previous step is the quantization of the Noether momenta.

The structure of the paper is as follows. In the next Section 2 we present the main characteristics of the method of quantization of Hamiltonian systems with a PDM by making use of Killing vector fields and Noether momenta. The rest of the paper is devoted to illustrate this method with some different particular systems. In Section 3 a nonlinear oscillator with quasi-harmonic behaviour is studied. In Section 4 we study the quantization of three nonlinear oscillators with a position-dependent mass and in Section 5 we consider the relation with the Laplace-Beltrami quantization formalism. We conclude in the last section with some remarks and open problems.

\section{Quantization by making use of Killing vector fields and Noether momenta}

\subsection{Killing vector fields and Noether momenta}

In order to study a quantum system (in the Schrödinger picture) we should first fix the Hilbert space $\mathcal{H}$ and then the (essentially) selfadjoint operators corresponding to the relevant observables to be quantized. Recall that there are obstructions for the quantization of all classical observables (see e.g. [41]), and sometimes we are only interested in the explicit form of the Hamiltonian quantum operator.

The domain of quantum operators is quite important in the non-bounded case. So, the selfadjoint character of an operator depends not only of the formal appearance of the operator, but also of the particular domain of the Hilbert space in which it is defined. The same formal aspect of an operator can lead to a selfadjoint operator in a case, or not selfadjoint in the other. Therefore the quantization of the Hamiltonian of a system means two items:

(a) Definition of the appropriate Hilbert space of pure states.

(b) Construction of the quantum Hamiltonian (defined in the Hilbert space (a)).

Unfortunately many authors go directly to the point (b) without a previous detailed analysis of the point (a). In the problem we are going to consider (quantization of a Hamiltonian system with a PDM) the point (a) is of a great importance because the particular form of the measure $d \mu$ defining the Hilbert space $L^{2}(\mathbb{R}, d \mu)$ strongly depends on the characteristics of the function $m(x)$.

Let us begin by considering the classical one-dimensional free-particle motion in the real line characterized by the $x$-dependent kinetic term $T$ as a Lagrangian

$$
L(x, v)=T(x, v)=\frac{1}{2} m(x) v^{2}, \quad m(x)>0,
$$


that leads to the following nonlinear differential equation

$$
m(x) \ddot{x}+\frac{1}{2} m^{\prime}(x) \dot{x}^{2}=0,
$$

where $m^{\prime}(x)=d m / d x$. As indicated in [42] this kinetic Lagrangian possesses an exact Noether symmetry. In fact, the function $T$ is not invariant under translations but under the action of the vector field $X$ given by

$$
X(x)=\frac{1}{\sqrt{m(x)}} \frac{\partial}{\partial x},
$$

(displacement $\delta x=\epsilon(m(x))^{-1 / 2}$, in the physicists language) in the sense that we have

$$
X^{t}(T)=0,
$$

where $X^{t}$ denotes the tangent lift to the velocity phase space $\mathbb{R} \times \mathbb{R}$ (that, in differential geometric terms, is the tangent bundle $T Q$ of the configuration space $Q=\mathbb{R})$ of the vector field $X \in \mathfrak{X}(\mathbb{R})$,

$$
X^{t}(x, v)=\frac{1}{\sqrt{m(x)}}\left(\frac{\partial}{\partial x}-\left(\frac{1}{2} \frac{m^{\prime}(x)}{m(x)}\right) v \frac{\partial}{\partial v}\right) .
$$

At this point we recall that given a Riemannian space $(M, g)$, with local coordinates $x^{1}, x^{2}, \ldots, x^{n}$, then a vector field $X$ defined on $M$ that is a symmetry of the metric $g$ (in the sense that it satisfies $\mathcal{L}_{X} g=0$ where $\mathcal{L}_{X}$ denotes the Lie derivative with respect to $\left.X\right)$ is called Killing vector field. We also recall that Killing vector fields also preserve the volume $\Omega_{g}$ determined by the metric, that is,

$$
\Omega_{g}=\sqrt{|g|} d x^{1} \wedge d x^{2} \wedge \ldots \wedge d x^{n}, \quad \mathcal{L}_{X} \Omega_{g}=0,
$$

where $|g|$ denotes the determinant of the matrix $g$ defining the Riemann structure.

The following proposition relates geometry with mechanics.

Proposition 1 Let $(M, g)$ a Riemannian space, $X$ a vector field on $M, X^{t}$ the tangent lift of $X$ to $T M$, and $T_{g}$ the kinetic energy function defined by the metric

$$
T_{g}(x, v)=\frac{1}{2} g_{i j}(x) v^{i} v^{j} .
$$

Then the important property is true

$$
X^{t}\left(T_{g}\right)=T_{\widetilde{g}}, \quad \widetilde{g}=\mathcal{L}_{X} g .
$$

For a proof of this proposition see [43].

Consequently, $X$ is a Killing vector field for the Riemann structure $g$ if and only if $X^{t}$ is a symmetry for the associated kinetic energy function $T_{g}$. Now, we can observe that the vector field $X$ given by (2.2), that preserve the PDM kinetic term (2.1), is in fact a Killing vector field of the one-dimensional $m$-dependent metric

$$
g=m(x) d x \otimes d x, \quad d s^{2}=m(x) d x^{2} .
$$

The line element is invariant under the flow of the vector field $X=f(x) \partial / \partial x$ when

$$
f m^{\prime}+2 m f^{\prime}=0,
$$


and, therefore, in order to the vector field $X$ to be a Killing vector, it should be proportional to the vector field $X$ given by (2.2).

The vector field $X$ represents (the infinitesimal generator of) an exact Noether symmetry for the geodesic motion. If we denote by $\theta_{L}$ the Lagrangian 1 -form

$$
\theta_{L}=\left(\frac{\partial L}{\partial v}\right) d x=m(x) v d x
$$

then the associated Noether constant of the motion $P$ for the free (geodesic) motion is given by

$$
P=i\left(X^{t}\right) \theta_{L}=\sqrt{m(x)} v .
$$

In what follows the function $P$ will be called Noether momentum associated to the Noether symmetry determined by $X$.

\subsection{Quasi-regular representation}

The Hilbert space for a quantum system with a classical configuration space $M$ is the linear space of square integrable functions on $M$ with respect to an appropriate measure, $L^{2}(M, d \mu)$. In the case of a natural system the measure to be considered must be invariant under the the Killing vector fields of the metric. The reason is the following:

If $\Phi: G \times M \rightarrow M$ denotes the action of a Lie group $G$ on a differentiable manifold $M$, then the associated quasi-regular representation is given by the following action of $G$ on the set of complex functions on $M$ :

$$
(U(g) \psi)(x)=\psi\left(\Phi\left(g^{-1}, x\right)\right) .
$$

If $M$ admits an invariant measure $d \mu$ we can restrict the action on the set $L^{2}(M, d \mu)$ and the linear representation so obtained is a unitary representation, because then

$$
\left\langle U(g) \psi_{1}, U(g) \psi_{2}\right\rangle=\int_{M}\left(\left(U(g) \psi_{1}\right)(x)\right)^{*}\left(U(g) \psi_{2}\right)(x) d \mu(x)
$$

i.e.

$$
\left\langle U(g) \psi_{1}, U(g) \psi_{2}\right\rangle=\int_{M}\left(\psi_{1}\left(\Phi\left(g^{-1}, x\right)\right)\right)^{*} \psi_{2}\left(\Phi\left(g^{-1}, x\right)\right) d \mu(x),
$$

and consequently, defining $y$ as $y=\Phi\left(g^{-1}, x\right)$, we obtain

$$
\left\langle U(g) \psi_{1}, U(g) \psi_{2}\right\rangle=\int_{M}\left(\psi_{1}(y)\right)^{*} \psi_{2}(y) d \mu(\Phi(g, y))=\int_{M}\left(\psi_{1}(y)\right)^{*} \psi_{2}(y) d \mu(y)=\left\langle\psi_{1}, \psi_{2}\right\rangle .
$$

If a one-parameter subgroup $\gamma(t)=\exp (a t), a \in \mathfrak{g}$, is considered, then the fundamental vector field $X_{a} \in \mathfrak{g}$, which is given by

$$
(X \psi)(x)=\frac{d}{d t} \psi(\Phi(\exp (-t a), x))_{\mid t=0},
$$

when restricted to the subspace $L^{2}(M, d \mu)$ is a skew-selfadjoint operator provided that the measure $\mu$ is $\gamma(t)$-invariant, because $U(\gamma(t))$ is a one-parameter group of unitary transformations. The infinitesimal generator in the regular representation is a generator for a 1-parameter group of unitary transformations, and consequently it is skew-self-adjoint operator. Of course if we want the generators of several oneparameter groups be skew-self-adjoint, the measure defining the Hilbert space must be invariant under each 1-parameter subgroup. 


\subsection{Quantization}

Coming back to the one-dimensional PDM system, the quantum system must be described by the Hilbert space of square integrable functions defined in $\mathbb{R}$ endowed with an invariant under $X$ measure, $d \mu_{x}$, therefore determined by the metric. The Lebesgue measure $d x$ is not invariant under $X=f(x) \partial / \partial x$, the invariance condition for the measure $d \mu=\rho(x) d x$ being

$$
f \rho^{\prime}+\rho f^{\prime}=0 .
$$

Therefore the only measure invariant under $X$ given by (2.2) is any multiple of

$$
d \mu_{x}=\sqrt{m(x)} d x .
$$

This automatically implies that the first-order linear operator $X$ is skew-symmetric. This means that the operator $\widehat{P}$ representing the quantum version of the Noether momentum $P$ must be selfadjoint, not in the standard space $L^{2}(\mathbb{R}) \equiv L^{2}(\mathbb{R}, d x)$, but in the space $L^{2}\left(\mathbb{R}, d \mu_{x}\right)$ of square integrable functions with respect the PDM measure $d \mu_{x}$.

Using the Legendre transformation the momentum $p$ and velocity $v$ are related by $p=m(x) v$, so that the expressions of the Noether momenta and the Hamiltonian (kinetic term plus a potential) in the phase space are

$$
P=\frac{1}{\sqrt{m(x)}} p
$$

and

$$
H=\frac{1}{2} P^{2}+V(x)
$$

As we have pointed out, the generator of the infinitesimal 'translation' symmetry, $(1 / \sqrt{m(x)}) d / d x$, is skew-Hermitian in the space $L^{2}\left(\mathbb{R}, d \mu_{x}\right)$ and therefore the transition from the classical system to the quantum one is given by defining the operator $\widehat{P}$ as follows

$$
P \mapsto \widehat{P}=\frac{1}{\sqrt{m(x)}}\left(-i \hbar \frac{d}{d x}\right)
$$

so that

$$
\frac{1}{m} p^{2} \rightarrow-\hbar^{2}\left(\frac{1}{\sqrt{m(x)}} \frac{d}{d x}\right)\left(\frac{1}{\sqrt{m(x)}} \frac{d}{d x}\right),
$$

in such a way that the quantum Hamiltonian $\widehat{H}$ is represented by the following Hermitian (defined on the space $\left.L^{2}\left(\mathbb{R}, d \mu_{x}\right)\right)$ operator

$$
\begin{aligned}
\widehat{H} & =-\frac{\hbar^{2}}{2}\left(\frac{1}{\sqrt{m(x)}} \frac{d}{d x}\right)\left(\frac{1}{\sqrt{m(x)}} \frac{d}{d x}\right)+V(x), \\
& =-\frac{\hbar^{2}}{2} \frac{1}{m(x)} \frac{d^{2}}{d x^{2}}+\frac{\hbar^{2}}{4}\left(\frac{m^{\prime}(x)}{m^{2}(x)}\right) \frac{d}{d x}+V(x),
\end{aligned}
$$

and then the Schrödinger equation $\widehat{H} \Psi=E \Psi$ becomes

$$
-\frac{\hbar^{2}}{2} \frac{1}{m(x)} \frac{d^{2} \Psi}{d x^{2}}+\frac{\hbar^{2}}{4}\left(\frac{m^{\prime}(x)}{m^{2}(x)}\right) \frac{d \Psi}{d x}+V(x) \Psi=E \Psi .
$$


We can summarize the method we have presented by emphasizing two important changes with respect the standard method of quantizing in the normal case of a constant mass. First, the Hilbert space is now related with a $m(x)$-dependent measure. Second, this method quantizes, not the canonical momenta $p$, but the Noether momenta $P$. Then the method is carried out in two steps:

(i) Study of the properties of the classical free particle with PDM (kinetic term without potential) using geometric techniques as an approach

Kinetic term $T \longrightarrow$ Killing vector field $X \longrightarrow$ Noether momentum $P$

(ii) Quantization (making use of the measure $d \mu_{x}$ ) first of $P$ and then of $H$

$$
\text { Noether momentum } P \longrightarrow \text { Hermitian operator } \widehat{P} \longrightarrow \text { Quantum Hamiltonian } \widehat{H}
$$

In the following Sections, we will illustrate this method of quantization with a detailed study of some particular cases. We will focus our attention on some systems related with nonlinear versions of the harmonic oscillator.

\section{Nonlinear quasi-harmonic oscillator with a PDM}

As a first example we review the quantization of a nonlinear oscillator already studied in [39, 40]. The nonlinear differential equation

$$
\left(1+\lambda x^{2}\right) \ddot{x}-(\lambda x) \dot{x}^{2}+\alpha^{2} x=0, \quad \lambda>0,
$$

was first studied by Mathews and Lakshmanan in [44. (see also [45]) as an example of a non-linear oscillator; the most remarkable property is that its general solution is of the form

$$
x=A \sin (\omega t+\phi), \quad \omega^{2}=\frac{\alpha^{2}}{1+\lambda A^{2}}, \quad \lambda>0 .
$$

That is, the above equation represents a non-linear oscillator with periodic solutions having a simple harmonic form. It can be proved that (3.1) can be obtained from the Euler-Lagrange equation of the Lagrangian

$$
L=\frac{1}{2}\left(\frac{1}{1+\lambda x^{2}}\right)\left(\dot{x}^{2}-\alpha^{2} x^{2}\right) \text {. }
$$

The generalization of this system to $n=2$ and $n>2$ dimensions (and also for both $\lambda>0$ and $\lambda<0$ ) was studied in [46. Since then it has been studied by different authors [47-58].

Let us consider the following Lagrangian

$$
L(x, v ; \kappa)=\frac{1}{2}\left(\frac{v^{2}}{1-\kappa x^{2}}\right)-\left(\frac{1}{2}\right) \alpha^{2}\left(\frac{x^{2}}{1-\kappa x^{2}}\right),
$$

where use has been made of a change of sign, $\kappa=-\lambda$ (the reason for this change is that, in the generalized higher-dimensional case, the new parameter $\kappa$ can be interpreted as a constant curvature), and the $\kappa$ dependence is defined in such a way that the limit when $\kappa \rightarrow 0$ is correctly defined and it leads to 
the linear harmonic oscillator. It is a system with a PDM $m=m(x)=1 /\left(1-\kappa x^{2}\right)$ that depends on the position $x$. Remark that when $\kappa>0$ we must restrict the configuration space to the open interval $(-1 / \sqrt{\kappa}, 1 / \sqrt{\kappa})$ in order to the mass be positive and to avoid singularities.

The $\kappa$-dependent kinetic term

$$
T_{\kappa}(x, v)=\frac{1}{2}\left(\frac{v^{2}}{1-\kappa x^{2}}\right)
$$

is invariant under the action of the vector field $X_{\kappa}$ given by

$$
X_{\kappa}(x)=\sqrt{1-\kappa x^{2}} \frac{\partial}{\partial x},
$$

in the sense that we have

$$
X_{\kappa}^{t}\left(T_{\kappa}\right)=0,
$$

where $X_{\kappa}^{t}$ denotes the natural lift to the velocity phase space (tangent bundle $T Q$ of the configuration space $Q$ ) of the vector field $X_{\kappa}$ in the configuration space,

$$
X_{\kappa}^{t}(x, v)=\sqrt{1-\kappa x^{2}} \frac{\partial}{\partial x}-\left(\frac{\kappa x v_{x}}{\sqrt{1-\kappa x^{2}}}\right) \frac{\partial}{\partial v_{x}} .
$$

In differential geometric terms this property means that the vector field $X_{\kappa}$ is a Killing vector field of the one-dimensional metric

$$
g=\left(\frac{1}{1-\kappa x^{2}}\right) d x \otimes d x, \quad d s_{\kappa}^{2}=\left(\frac{1}{1-\kappa x^{2}}\right) d x^{2} .
$$

It must also be considered as a Noether symmetry for the geodesic motion with an associated Noether constant of the motion $P$ for the geodesic motion that is given by

$$
P=i\left(X^{t}\right) \theta_{L}=\left(\frac{1}{\sqrt{1-\kappa x^{2}}}\right) v .
$$

The expression of $P$ in the phase space is

$$
P=\sqrt{1-\kappa x^{2}} p_{x}
$$

so that the (classical) Hamiltonian of this $\kappa$-dependent oscillator can be written as follows

$$
H=\left(\frac{1}{2}\right) P^{2}+\left(\frac{1}{2}\right) \alpha^{2}\left(\frac{x^{2}}{1-\kappa x^{2}}\right) .
$$

The quantum formalism is constructed by considering wave functions on the real line $\mathbb{R}$ (when $\kappa<0$ ) or in the interval $(-1 / \sqrt{\kappa}, 1 / \sqrt{\kappa})$, (if $\kappa>0)$, endowed with the measure $d \mu_{\kappa}$ given by

$$
d \mu_{\kappa}=\left(\frac{1}{\sqrt{1-\kappa x^{2}}}\right) d x
$$

which is the only (up to a factor) measure invariant under $X_{\kappa}$. This means that the operator $\widehat{P}$, representing the PDM linear momentum, must be Hermitian in the space $L^{2}\left(d \mu_{\kappa}\right)$ of square integrable functions with respect the PDM measure $d \mu_{\kappa}$ defined as 
(i) In the negative $\kappa<0$ case, the space $L^{2}\left(d \mu_{\kappa}\right)$ is $L^{2}\left(\mathbb{R}, d \mu_{\kappa}\right)$.

(ii) In the positive $\kappa>0$ case, the space $L^{2}\left(d \mu_{\kappa}\right)$ is $L_{0}^{2}\left(I_{\kappa}, d \mu_{\kappa}\right)$ where $I_{\kappa}$ denotes the interval $[-\sqrt{\kappa}, 1 / \sqrt{\kappa}]$ and the subscript means that the functions must vanish at the endpoints $x=-1 / \sqrt{\kappa}$ and $x=1 / \sqrt{\kappa}$.

(iii) Of course in the $\kappa=0$ case we recover the standard space $L^{2}(\mathbb{R}, d x)$.

The quantization is given by

$$
P \mapsto \widehat{P}=-i \hbar \sqrt{1-\kappa x^{2}} \frac{d}{d x}
$$

so that

$$
\left(1-\kappa x^{2}\right) p_{x}^{2} \rightarrow-\hbar^{2}\left(\sqrt{1-\kappa x^{2}} \frac{d}{d x}\right)\left(\sqrt{1-\kappa x^{2}} \frac{d}{d x}\right),
$$

in such a way that the quantum version $\widehat{H}$ of the Hamiltonian $H$ becomes

$$
\widehat{H}=-\frac{\hbar^{2}}{2}\left(1-\kappa x^{2}\right) \frac{d^{2}}{d x^{2}}+\left(\frac{\hbar^{2}}{2}\right) \kappa x \frac{d}{d x}+\left(\frac{1}{2}\right) \alpha^{2}\left(\frac{x^{2}}{1-\kappa x^{2}}\right) .
$$

Finally, introducing dimensionless variables $(\widetilde{x}, \widetilde{\kappa}, e)$

$$
x=\left(\sqrt{\frac{\hbar}{\alpha}}\right) \widetilde{x}, \quad \kappa=\left(\frac{\alpha}{\hbar}\right) \widetilde{\kappa}, \quad E=(\hbar \alpha) e,
$$

we arrive to

- The quantum Hamiltonian $\widehat{H}$ becomes

$$
\widehat{H}=\left[-\frac{1}{2}\left(1-\widetilde{\kappa} \widetilde{x}^{2}\right) \frac{d^{2}}{d \widetilde{x}^{2}}+\left(\frac{1}{2}\right) \widetilde{\kappa} \widetilde{x} \frac{d}{d \widetilde{x}}+\left(\frac{1}{2}\right)\left(\frac{\widetilde{x}^{2}}{1-\widetilde{\kappa} \widetilde{x}^{2}}\right)\right](\hbar \alpha) .
$$

- The Schrödinger equation reduces to the following dimensionless form

$$
\left(1-\widetilde{k} \widetilde{x}^{2}\right) \frac{d^{2}}{d \widetilde{x}^{2}} \Psi-\widetilde{k} \widetilde{x} \frac{d}{d \widetilde{x}} \Psi-\left(\frac{\widetilde{x}^{2}}{1-\widetilde{\kappa} \widetilde{x}^{2}}\right) \Psi+(2 e) \Psi=0 .
$$

\section{Three nonlinear oscillators with a position-dependent mass}

In this section we consider three particular one-dimensional nonlinear oscillators with a position dependent mass (PDM). They were studied in [14] with the formalism of creation-annihilation operators; now we study the quantization by making use of the method presented in Section (2) as an approach.

\section{$4.1 \lambda$-dependent nonlinear oscillator no. 1}

The position dependent mass $m_{1}$ and the potential $V_{1}$ are

$$
m_{1}=\frac{m_{0}}{\left(1+\lambda^{2} x^{2}\right)} \quad \text { and } \quad V_{1}=\left(\frac{m_{0}}{2 \lambda^{2}}\right) \alpha^{2}\left(\operatorname{arcsinh}^{2}(\lambda x)\right), \quad m_{0}>0
$$


and therefore the Lagrangian is given by

$$
L_{1}(x, v ; \lambda)=T_{1 \lambda}(x, v ; \lambda)-V_{1}(x ; \lambda)=\frac{1}{2} m_{0}\left(\frac{v^{2}}{1+\lambda^{2} x^{2}}\right)-\left(\frac{m_{0}}{2 \lambda^{2}}\right) \alpha^{2}\left(\operatorname{arcsinh}^{2}(\lambda x)\right),
$$

where $\lambda$ is a parameter such that $\lambda x$ is dimensionless. The $\lambda$ dependence is defined in such a way that the following limit is satisfied

$$
\lim _{\lambda \rightarrow 0} L_{1}(x, v ; \lambda)=\frac{1}{2} m_{0} v^{2}-\frac{1}{2} m_{0} \alpha^{2} x^{2} .
$$

The kinetic term $T_{1 \lambda}$ is invariant under the action of the vector field $X_{\lambda}$ given by

$$
X_{\lambda}(x)=\sqrt{1+\lambda^{2} x^{2}} \frac{\partial}{\partial x},
$$

in the sense that we have

$$
X_{\lambda}^{t}\left(T_{1 \lambda}\right)=0,
$$

where $X_{\lambda}^{t}$ denotes the natural lift to the velocity phase space $\mathbb{R} \times \mathbb{R}$ (tangent bundle $T Q$ of the configuration space $Q=\mathbb{R}$ ) of the vector field $X_{\lambda}$,

$$
X_{\lambda}^{t}(x, v)=\sqrt{1+\lambda^{2} x^{2}} \frac{\partial}{\partial x}+\left(\frac{\lambda^{2} x v}{\sqrt{1+\lambda^{2} x^{2}}}\right) \frac{\partial}{\partial v} .
$$

In differential geometric terms this property means that the vector field $X_{\lambda}$ is a Killing vector field of the one-dimensional metric

$$
g=\left(\frac{1}{1+\lambda^{2} x^{2}}\right) d x \otimes d x, \quad d s_{\lambda}^{2}=\left(\frac{1}{1+\lambda^{2} x^{2}}\right) d x^{2},
$$

and, from a dynamial viewpoint, $X_{\lambda}$ must be considered as a Noether symmetry for the geodesic motion. The associated Noether constant of the motion $P$ for such geodesic motion is given by

$$
P=i\left(X_{\lambda}^{t}\right) \theta_{L}=\left(\frac{m_{0}}{\sqrt{1+\lambda^{2} x^{2}}}\right) v .
$$

From this result we obtain that the (classical) Hamiltonian of this $\lambda$-dependent oscillator can be written as follows

$$
H_{1}=\left(\frac{1}{2 m_{0}}\right) P^{2}+\left(\frac{m_{0}}{2 \lambda^{2}}\right) \alpha^{2} \operatorname{arcsinh}^{2}(\lambda x), \quad P=\sqrt{\left(1+\lambda^{2} x^{2}\right)} p .
$$

The quantum formalism is constructed with wave functions defined on the real line $\mathbb{R}$ endowed with the measure $d \mu_{\lambda}$ given by

$$
d \mu_{\lambda}=\left(\frac{1}{\sqrt{1+\lambda^{2} x^{2}}}\right) d x
$$

which is the particular measure determined by the metric and also the only (up to a constant factor) measure invariant under $X_{\lambda}$. This means that the operator $\widehat{P}$, representing the PDM linear momentum, must be Hermitian, not in the standard space $L^{2}(\mathbb{R})$, but in the space $L^{2}\left(\mathbb{R}, d \mu_{\lambda}\right)$; of course $L^{2}\left(\mathbb{R}, d \mu_{\lambda}\right)$ reduces in the limit $\lambda \rightarrow 0$ to $L^{2}(\mathbb{R}, d x) \equiv L^{2}(\mathbb{R})$. The correspondence $P \rightarrow \widehat{P}$ is given by

$$
P \mapsto \widehat{P}=-i \hbar \sqrt{1+\lambda^{2} x^{2}} \frac{d}{d x},
$$


so that

$$
\left(1+\lambda^{2} x^{2}\right) p^{2} \rightarrow P^{2} \rightarrow-\hbar^{2}\left(\sqrt{1+\lambda^{2} x^{2}} \frac{d}{d x}\right)\left(\sqrt{1+\lambda^{2} x^{2}} \frac{d}{d x}\right)
$$

in such a way that the quantum version $\widehat{H_{1}}$ of the Hamiltonian $H_{1}$ is

$$
\widehat{H_{1}}=-\frac{\hbar^{2}}{2 m_{0}}\left(1+\lambda^{2} x^{2}\right) \frac{d^{2}}{d x^{2}}-\left(\frac{\hbar^{2}}{2 m_{0}}\right) \lambda^{2} x \frac{d}{d x}+\left(\frac{m_{0}}{2 \lambda^{2}}\right) \alpha^{2}\left(\operatorname{arcsinh}^{2}(\lambda x)\right) .
$$

and then the Schrödinger equation

$$
\widehat{H_{1}} \Psi=E \Psi
$$

turns out to be

$$
\left[-\frac{\hbar^{2}}{2 m_{0}}\left(1+\lambda^{2} x^{2}\right) \frac{d^{2}}{d x^{2}}-\left(\frac{\hbar^{2}}{2 m_{0}}\right) \lambda^{2} x \frac{d}{d x}+\left(\frac{m_{0}}{2 \lambda^{2}}\right) \alpha^{2}\left(\operatorname{arcsinh}^{2}(\lambda x)\right)\right] \Psi=e(\hbar \alpha) \Psi .
$$

It is convenient to simplify this equation by introducing dimensionless variables $(\widetilde{x}, \Lambda, e)$ defined by

$$
x=\left(\sqrt{\frac{\hbar}{m_{0} \alpha}}\right) \widetilde{x}, \quad \lambda=\left(\sqrt{\frac{m_{0} \alpha}{\hbar}}\right) \Lambda, \quad E=(\hbar \alpha) e,
$$

in such a way that then

- The quantum Hamiltonian $\widehat{H_{1}}$ becomes

$$
\widehat{H_{1}}=\left[-\frac{1}{2}\left(1+\Lambda^{2} \widetilde{x}^{2}\right) \frac{d^{2}}{d \widetilde{x}^{2}}-\left(\frac{1}{2}\right) \Lambda^{2} \widetilde{x} \frac{d}{d \widetilde{x}}+\left(\frac{1}{2 \Lambda^{2}}\right) \operatorname{arcsinh}^{2}(\Lambda \widetilde{x})\right](\hbar \alpha) .
$$

- The Schrödinger equation reduces, in terms of dimensionless variables, to the following form:

$$
\left(1+\Lambda^{2} \widetilde{x}^{2}\right) \frac{d^{2}}{d \widetilde{x}^{2}} \Psi+\Lambda^{2} \widetilde{x} \frac{d}{d \widetilde{x}} \Psi-\left(\frac{1}{\Lambda^{2}}\right)\left(\operatorname{arcsinh}^{2}(\Lambda \widetilde{x})\right) \Psi+(2 e) \Psi=0 .
$$

\section{$4.2 \quad \lambda$-dependent nonlinear oscillator no. 2}

The position dependent mass $m_{2}$ and the potential $V_{2}$ are

$$
m_{2}=\frac{m_{0}}{(1+\lambda x)^{2}} \quad \text { and } \quad V_{2}(x ; \lambda)=\left(\frac{m_{0}}{2 \lambda^{2}}\right) \alpha^{2}\left(\log ^{2}(1+\lambda x)\right),
$$

where we restrict the configuration space to the interval $(-1 / \lambda, \infty)$. Therefore the Lagrangian, that is given by

$$
L_{2}(x, v ; \lambda)=T_{2 \lambda}(x, v ; \lambda)-V_{2}(x ; \lambda)=\frac{1}{2} m_{0}\left(\frac{v^{2}}{(1+\lambda x)^{2}}\right)-\left(\frac{m_{0}}{2 \lambda^{2}}\right) \alpha^{2}\left(\log ^{2}(1+\lambda x)\right),
$$

is correctly defined in $I_{\lambda}=(-1 / \lambda, \infty)$ and also in this case the following limit is satisfied

$$
\lim _{\lambda \rightarrow 0} L_{2}(x, v ; \lambda)=\frac{1}{2} m_{0} v^{2}-\frac{1}{2} m_{0} \alpha^{2} x^{2} .
$$


The kinetic term $T_{2 \lambda}$ is invariant under the action of the vector field $X_{\lambda}$ given by

$$
X_{\lambda}(x)=(1+\lambda x) \frac{\partial}{\partial x},
$$

in the sense that we have

$$
X_{\lambda}^{t}\left(T_{2 \lambda}\right)=0,
$$

where $X_{\lambda}^{t}$ denotes the natural lift to the velocity phase space $\mathbb{R} \times I_{\lambda}$ (tangent bundle $T Q$ of $Q=I_{\lambda}$ ) of the vector field $X_{\lambda}$,

$$
X_{\lambda}^{t}(x, v)=(1+\lambda x) \frac{\partial}{\partial x}+\lambda v \frac{\partial}{\partial v} .
$$

In differential geometric terms this property means that the vector field $X_{\lambda}$ is a Killing vector field of the one-dimensional metric

$$
g=\left(\frac{1}{(1+\lambda x)^{2}}\right) d x \otimes d x, \quad d s_{\lambda}^{2}=\left(\frac{1}{(1+\lambda x)^{2}}\right) d x^{2} .
$$

It must also be considered as a Noether symmetry for the geodesic motion. The associated Noether constant of the motion $P$ for the geodesic motion is given by

$$
P=i\left(X_{\lambda}^{t}\right) \theta_{L}=\left(\frac{m_{0}}{(1+\lambda x)}\right) v,
$$

so that the (classical) Hamiltonian of this $\lambda$-dependent oscillator can be written as follows

$$
H_{2}=\left(\frac{1}{2 m_{0}}\right) P^{2}+\left(\frac{m_{0}}{\lambda^{2}}\right)\left(\log ^{2}(1+\lambda x)\right), \quad P=(1+\lambda x) p .
$$

The quantum formalism is constructed with wave functions defined on the interval $I_{\lambda}=(-1 / \lambda, \infty)$ endowed with the measure $d \mu_{\lambda}$ given by

$$
d \mu_{\lambda}=\left(\frac{1}{(1+\lambda x)}\right) d x
$$

which is the measure determined by the one-dimensional metric and it is the only (up to a constant factor) measure invariant under $X_{\lambda}$. Note also that in the limit for $\lambda \rightarrow 0, d \mu_{\lambda}$ reduces to $d x$. This means that the operator $\widehat{P}$, representing the PDM linear momentum, must be Hermitian, not in the standard space $L^{2}\left(I_{\lambda}\right)$ with $I_{\lambda}=(-1 / \lambda, \infty)$, but in the space $L_{0}^{2}\left(I_{\lambda}, d \mu_{\lambda}\right)$ with $d \mu_{\lambda}$ as defined above (the subscript means that the functions must vanish at the point $x=-1 / \lambda)$. The transition $P \rightarrow \widehat{P}$ is given by

$$
P \mapsto \widehat{P}=-i \hbar(1+\lambda x) \frac{d}{d x}
$$

so that

$$
(1+\lambda x)^{2} p^{2} \rightarrow-\hbar^{2}\left((1+\lambda x) \frac{d}{d x}\right)\left((1+\lambda x) \frac{d}{d x}\right),
$$

in such a way that the quantum version $\widehat{H_{2}}$ of the Hamiltonian $H_{2}$ becomes

$$
\widehat{H_{2}}=-\frac{\hbar^{2}}{2 m_{0}}(1+\lambda x)^{2} \frac{d^{2}}{d x^{2}}-\left(\frac{\hbar^{2}}{2 m_{0}}\right) \lambda(1+\lambda x) \frac{d}{d x}+\left(\frac{m_{0}}{2 \lambda^{2}}\right) \alpha^{2}\left(\log ^{2}(1+\lambda x)\right),
$$


and then the Schrödinger equation

$$
\widehat{H_{2}} \Psi=E \Psi
$$

is

$$
\left[-\frac{\hbar^{2}}{2 m_{0}}(1+\lambda x)^{2} \frac{d^{2}}{d x^{2}}-\left(\frac{\hbar^{2}}{2 m_{0}}\right) \lambda(1+\lambda x) \frac{d}{d x}+\left(\frac{m_{0}}{2 \lambda^{2}}\right) \alpha^{2}\left(\log ^{2}(1+\lambda x)\right)\right] \Psi=e \Psi,
$$

It is convenient to simplify this equation by introducing dimensionless variables $(\widetilde{x}, \Lambda, e)$ defined by

$$
x=\left(\sqrt{\frac{\hbar}{m_{0} \alpha}}\right) \widetilde{x}, \quad \lambda=\left(\sqrt{\frac{m_{0} \alpha}{\hbar}}\right) \Lambda, \quad E=(\hbar \alpha) e,
$$

in such a way that $\lambda x=\Lambda \widetilde{x}$ and then we see that:

- The quantum Hamiltonian $\widehat{H_{2}}$ becomes

$$
\widehat{H_{2}}=\left[-\frac{1}{2}(1+\Lambda \widetilde{x})^{2} \frac{d^{2}}{d \widetilde{x}^{2}}-\left(\frac{1}{2}\right) \Lambda(1+\Lambda \widetilde{x}) \frac{d}{d \widetilde{x}}+\left(\frac{1}{2 \Lambda^{2}}\right) \log ^{2}(1+\Lambda \widetilde{x}](\hbar \alpha) .\right.
$$

- The Schrödinger equation reduces, in terms of dimensionless variables, to the following form:

$$
(1+\Lambda \widetilde{x})^{2} \frac{d^{2}}{d \widetilde{x}^{2}} \Psi+\Lambda(1+\Lambda \widetilde{x}) \frac{d}{d \widetilde{x}} \Psi-\left(\frac{1}{\Lambda^{2}}\right)\left(\log ^{2}(1+\Lambda \widetilde{x})\right) \Psi+(2 e) \Psi=0 .
$$

\section{3 $\lambda$-dependent nonlinear oscillator no. 3}

The position dependent mass $m_{3}$ and the potential $V_{3}$ are

$$
m_{3}=\frac{m_{0}}{\left(1-\lambda^{2} x^{2}\right)^{2}} \quad \text { and } \quad V_{3}(x ; \lambda)=\left(\frac{m_{0}}{2 \lambda^{2}}\right) \alpha^{2}\left(\operatorname{arctanh}^{2}(\lambda x)\right), \quad \lambda \geq 0,
$$

and therefore the Lagrangian $L_{3}$ is given by

$$
L_{3}(x, v ; \lambda)=T_{3 \lambda}(x, v ; \lambda)-V_{3}(x ; \lambda)=\frac{1}{2} m_{0}\left(\frac{v^{2}}{\left(1-\lambda^{2} x^{2}\right)^{2}}\right)-\left(\frac{m_{0}}{2 \lambda^{2}}\right) \alpha^{2}\left(\operatorname{arctanh}^{2}(\lambda x)\right),
$$

so the dynamics is only defined in $I_{\lambda}=(-1 / \lambda, 1 / \lambda)$ and also in this case the limit $\lambda \rightarrow 0$ is

$$
\lim _{\lambda \rightarrow 0} L_{3}(x, v ; \lambda)=\frac{1}{2} m_{0} v^{2}-\frac{1}{2} m_{0} \alpha^{2} x^{2} .
$$

The kinetic term $T_{3 \lambda}$ is invariant under the action of the vector field $X_{\lambda}$ given by

$$
X_{\lambda}(x)=\left(1-\lambda^{2} x^{2}\right) \frac{\partial}{\partial x},
$$

in the sense that we have

$$
X_{\lambda}^{t}\left(T_{3 \lambda}\right)=0,
$$

where $X_{\lambda}^{t}$ denotes the natural lift to the velocity phase space $\mathbb{R} \times I_{\lambda}$ (tangent bundle $T Q$ of $Q=I_{\lambda}$ ) of the vector field $X_{\kappa}$,

$$
X_{\lambda}^{t}(x, v)=\left(1-\lambda^{2} x^{2}\right) \frac{\partial}{\partial x}-2 \lambda^{2} x v \frac{\partial}{\partial v} .
$$


In differential geometric terms this property means that the vector field $X_{\lambda}$ is a Killing vector field of the one-dimensional metric

$$
g=\left(\frac{1}{\left(1-\lambda^{2} x^{2}\right)^{2}}\right) d x \otimes d x, \quad d s_{\lambda}^{2}=\left(\frac{1}{\left(1-\lambda^{2} x^{2}\right)^{2}}\right) d x^{2} .
$$

It must also be considered as a Noether symmetry for the geodesic motion. The associated Noether constant of the motion $P$ for the geodesic motion is given by

$$
P=i\left(X_{\lambda}^{t}\right) \theta_{L}=\left(\frac{m_{0}}{\left(1-\lambda^{2} x^{2}\right)}\right) v,
$$

and the (classical) Hamiltonian of this $\lambda$-dependent oscillator can be written as follows

$$
H_{3}=\left(\frac{1}{2 m_{0}}\right) P^{2}+\left(\frac{m_{0}}{2 \lambda^{2}}\right)\left(\operatorname{arctanh}^{2}(\lambda x)\right), \quad P=\left(1-\lambda^{2} x^{2}\right) p .
$$

The quantum formalism is constructed with wave functions defined on the interval $I_{\lambda}=(-1 / \lambda, 1 / \lambda)$ endowed with the measure $d \mu_{\lambda}$ given by

$$
d \mu_{\lambda}=\left(\frac{1}{\left(1-\lambda^{2} x^{2}\right)}\right) d x
$$

which is the measure determined by the one-dimensional metric and it is the only (up to a constant factor) measure invariant under $X_{\lambda}$. Note also that in the limit for $\lambda \rightarrow 0, d \mu_{\lambda}$ reduces to $d x$. This means that the operator $\widehat{P}$, representing the PDM linear momentum, must be Hermitian, not in the standard space $L^{2}\left(I_{\lambda}\right) \equiv L^{2}\left(I_{\lambda}, d x\right)$ with $I_{\lambda}=(-1 / \lambda, 1 / \lambda)$, but in the space $L_{0}^{2}\left(I_{\lambda}, d \mu_{\lambda}\right)$ (the subscript means that the functions must vanish at the endpoints $x=-1 / \lambda$ and $x=1 / \lambda)$. The transition $P \rightarrow \widehat{P}$ is given by

$$
P \mapsto \widehat{P}=-i \hbar\left(1-\lambda^{2} x^{2}\right) \frac{d}{d x},
$$

so that

$$
\left(1+\lambda^{2} x^{2}\right) p^{2} \rightarrow-\hbar^{2}\left(\left(1-\lambda^{2} x^{2}\right) \frac{d}{d x}\right)\left(\left(1-\lambda^{2} x^{2}\right) \frac{d}{d x}\right),
$$

in such a way that the quantum version $\widehat{H_{3}}$ of the Hamiltonian $H_{3}$ is

$$
\widehat{H_{3}}=-\frac{\hbar^{2}}{2 m_{0}}\left(1-\lambda^{2} x^{2}\right)^{2} \frac{d^{2}}{d x^{2}}+\left(\frac{\hbar^{2}}{2 m_{0}}\right) 2 \lambda^{2} x\left(1-\lambda^{2} x^{2}\right) \frac{d}{d x}+\left(\frac{m_{0}}{2 \lambda^{2}}\right) \alpha^{2}\left(\operatorname{arctanh}^{2}(\lambda x)\right) .
$$

and then the Schrödinger equation turns out to be

$$
\widehat{H_{3}} \Psi=E \Psi
$$

becomes

$$
\left[-\frac{\hbar^{2}}{2 m_{0}}\left(1-\lambda^{2} x^{2}\right)^{2} \frac{d^{2}}{d x^{2}}+\left(\frac{\hbar^{2}}{2 m_{0}}\right) 2 \lambda^{2} x\left(1-\lambda^{2} x^{2}\right) \frac{d}{d x}+\left(\frac{m_{0}}{2 \lambda^{2}}\right) \alpha^{2}\left(\operatorname{arctanh}^{2}(\lambda x)\right)\right] \Psi=E \Psi .
$$

It is convenient to simplify this equation by introducing dimensionless variables $(\widetilde{x}, \Lambda, e)$ defined by

$$
x=\left(\sqrt{\frac{\hbar}{m_{0} \alpha}}\right) \widetilde{x}, \quad \lambda=\left(\sqrt{\frac{m_{0} \alpha}{\hbar}}\right) \Lambda, \quad E=(\hbar \alpha) e,
$$

in such a way that then 
- The quantum Hamiltonian $\widehat{H_{3}}$ becomes

$$
\widehat{H_{3}}=\left[-\frac{1}{2}\left(1-\Lambda^{2} \widetilde{x}^{2}\right)^{2} \frac{d^{2}}{d \widetilde{x}^{2}}-\left(\frac{1}{2}\right) 2 \lambda^{2} \widetilde{x}\left(1-\Lambda^{2} \widetilde{x}^{2}\right) \frac{d}{d \widetilde{x}}+\left(\frac{1}{2 \Lambda^{2}}\right) \operatorname{arctanh}^{2}(\Lambda \widetilde{x})\right](\hbar \alpha) .
$$

- The Schrödinger equation reduces, in terms of dimensionless variables, to the following form:

$$
\left(1-\Lambda^{2} \widetilde{x}^{2}\right)^{2} \frac{d^{2}}{d \widetilde{x}^{2}} \Psi-2 \lambda^{2} \widetilde{x}\left(1-\Lambda^{2} \widetilde{x}^{2}\right) \frac{d}{d \widetilde{x}} \Psi-\left(\frac{1}{\Lambda^{2}}\right)\left(\operatorname{arctanh}^{2}(\Lambda \widetilde{x})\right)+(2 e) \Psi=0 .
$$

\section{Relation with the Laplace-Beltrami quantization formalism}

It is known that the quantization rule $p_{i} \rightarrow \widehat{p_{i}}$, with the operator $\widehat{p_{i}}$ represented by the linear operator $\widehat{p_{i}}=-i \hbar\left(\partial / \partial x_{i}\right)$ is only correct when the configuration space $Q$ of the system is an Euclidean space (as for example $\mathbb{R}^{2}$ or $\mathbb{R}^{3}$ ) and then a classical Hamiltonian $H$, assumed of mechanical type, can be written as a function of the Cartesian-Rectangular coordinates $x_{i}$. Nevertheless, the momentum conjugate to an arbitrary generalized coordinate $q$ is, in general, not represented by $-i \hbar(\partial / \partial q)$. For example, if the classical Hamiltonian $H$ is presented in spherical coordinates $(r, \theta, \phi)$ then the quantization rule $p_{r} \rightarrow \widehat{p_{r}}$ with $\widehat{p_{r}}=-i \hbar(\partial / \partial r)$ is not correct [59].

In the Euclidean case, the kinetic term of the classical Hamiltonian is transformed into the Laplacian

$$
p_{x}^{2}+p_{y}^{2}+p_{z}^{2} \rightarrow \widehat{p}_{x}^{2}+\widehat{p}_{y}^{2}+\widehat{p}_{z}^{2}=-\hbar^{2} \nabla^{2}, \quad \nabla^{2}=\frac{\partial^{2}}{\partial x^{2}}+\frac{\partial^{2}}{\partial y^{2}}+\frac{\partial^{2}}{\partial z^{2}},
$$

and this fact suggests that the quantification of the kinetic term of a Hamiltonian written in curvilinear (non-Cartesian) coordinates is given by the Laplace operator in such coordinates [59]. In the nonEuclidean case, Schrödinger [60] and Stevenson 61] studied the spherical model (see also [62]) and Infeld and Shild 63 studied the corresponding problem in Lobachevsky plane (see also, e.g. 64, 65), and it is now well established that we can consider an analogous rule in Riemann spaces (see e.g. 66] and Section 2 of [67]).

So, if the configuration space $Q$ is endowed with a Riemann metric $g$ given by

$$
g=g_{i j}(q) d q^{i} \otimes d q^{j}, \quad d s^{2}=g_{i j}(q) d q^{i} d q^{j},
$$

the kinetic part of the quantum Hamiltonian is chosen to be given by

$$
\widehat{H}_{0}=-\frac{\hbar^{2}}{2 m} \nabla^{2}
$$

where $\nabla^{2}$ denotes the Laplace-Beltrami operator

$$
\nabla^{2} f=\operatorname{div}(\operatorname{grad} f)=\frac{1}{\sqrt{|g|}} \frac{\partial}{\partial q^{i}}\left(\sqrt{|g|}\left(g^{i j} \frac{\partial f}{\partial q^{j}}\right)\right) .
$$

That is, the Laplace-Beltrami quantization formalism quantizes directly the Hamiltonian (that is, $H \rightarrow$ $\widehat{H})$ without the previous quantization of the momenta. A particular example is that of the motion on a surface in $\mathbb{R}^{3}$, the metric been the pull-back of the Euclidean metric. 
As we have stated in Section (2), the kinetic term of a one-dimensional system with a PDM can be considered as associated to a one-dimensional metric $g$ with only one component

$$
g_{11}=m(x), g^{11}=\frac{1}{m(x)},|g|=g_{11},
$$

so that the one-dimensional version of the Laplace-Beltrami operator is given by

$$
\nabla^{2} f=\frac{1}{\sqrt{m(x)}} \frac{d}{d x}\left[\sqrt{m(x)}\left(\frac{1}{m} \frac{d f}{d x}\right)\right]=\frac{1}{m(x)} \frac{d^{2} f}{d x^{2}}-\frac{1}{2}\left(\frac{m^{\prime}(x)}{m^{2}(x)}\right) \frac{d f}{d x}
$$

and it leads to a Hamiltonian $\widehat{H}$ that takes the form

$$
\widehat{H}=-\frac{\hbar^{2}}{2} \frac{1}{m(x)} \frac{d^{2}}{d x^{2}}+\frac{\hbar^{2}}{4}\left(\frac{m^{\prime}(x)}{m^{2}(x)}\right) \frac{d}{d x}+V(x) .
$$

It is important to remark that it coincides with the Hamiltonian (2.4) obtained by making use of the quantization of the Noether momenta.

It is also to be remarked that it is known since the well known paper 68] that some quantum geometry induced potential terms related to curvatures can appear in some quantizations as constrained systems, but we can always absorbe such terms by defining a effective potential (see e.g. [69, 70, 171] or [67] for a more recent paper).

\section{Concluding remarks and outlook}

We have studied the quantization of Hamiltonian systems with a position-dependent mass by making use of the quantization of the Noether momenta (instead of the canonical momenta) as an approach. This means a first analysis of the PDM geodesic motion (motion with PDM but without potential) for obtaining the Noether momenta (integral of motion for the free particle but not for the total Hamiltonian). This is so because for a natural system the symplectic form only depends on the kinetic term and not of the potential term. In addition we have pointed out that the Hilbert space of wave functions must also depend on the PDM. Actually, only if an invariant measure is considered the operators obtained as generators of Killing transformations are Hermitean.

It is important to underline that this method, that represents an approach to the problem rather different to the formalism $(\alpha, \beta, \gamma)$ (see the references mentioned in Section (1.11)), is more than a simple practical recipe; it is in fact a method constructed on well defined mathematical bases. In addition, (as was proved in Section (5)), it leads to an expression of the quantum Hamiltonian that coincides with the one obtained by making use of the Laplace-Beltrami quantization formalism, a property which is not true anymore for the so called $(\alpha, \beta, \gamma)$ formalism. Nevertheless there is an important difference: the Laplace-Beltrami quantization formalism gives directly the expression of the Hamiltonian without the previous quantization of the momenta and the method we have studied is a two step quantization procedure (similar to the quantization of systems with constant mass): first quantization of momenta and then quantization of the Hamiltonian.

We finalize with the following comments. First, as we have mentioned in the Section (11), the interest for the Hamiltonian systems with PDM has increased in these last years; so the method we have studied must be applied to the quantization of all these systems (we have only considered some particular examples 
mainly related with the harmonic oscillator). Second, we have limited our study to one-dimensional systems but this method must be studied in the more general case of several degrees of freedom. Finally, the quantization rule $p \rightarrow \widehat{p}$, with the operator $\widehat{p}$ represented by the linear operator $\widehat{p}=-i \hbar \partial / \partial q$ is not valid either on non-Euclidean spaces, or on non-Cartesian coordinates. How to proceed then in a non-Euclidean one-dimensional systems, where Cartesian coordinates do not exist?. It seems natural to consider the quantization of systems defined on curved spaces by making use of the quantization of Noether momenta.

\section{Acknowledgments}

JFC and MFR acknowledge support from research projects MTM2015-64166-C2-1 (MINECO, Madrid) and DGA-E24/1 (DGA, Zaragoza) and MS from research projects MTM2014-57129-C2-1-P (MINECO, Madrid) and Junta de Castilla y León Project No. VA057U16.

\section{References}

[1] von Roos O., "Position dependent affective masses in semiconductor theory", Phys. Rev. B 27, 7547-7552 (1983).

[2] BenDaniel D.J., Duke C.B., "Space-charge effects on electron tunneling", Phys. Rev. 152, no. 2, $683-692$ (1966).

[3] Zhu Q.G., Kroemer H., "Interface connection rules for effective mass wave functions at an abrupt heterojunction between two different semiconductors", Phys. Rev. B 27, 3519-3527 (1983).

[4] Li T.L., Kuhn K., "Band-offset ratio dependence on the effective mass Hamiltonian based on a modified profile of the GaAsAlGa as a quantum well", Phys. Rev. B 47, no. 19, 12760-12770 (1993).

[5] de Souza Dutra A., Almeida C.A.S., "Exact solvability of potentials with spatially dependent effective masses", Phys. Lett. A 275, no. 1-2, 25-30 (2000).

[6] Roy B., Roy P., "A Lie algebraic approach to effective mass Schrödinger equations", J. Phys. A 35, 3961-3969 (2002).

[7] Koc R., Koca M., Korcuk E., "A new class of quasi-exactly solvable potentials with position dependent mass", J. Phys. A 35, L527-530 (2002).

[8] Quesne C., Tkachuk V.M., "Deformed algebras, position-dependent effective masses and curved spaces: an exactly solvable Coulomb problem", J. Phys. A 37, 4267-4281 (2004).

[9] Bagchi B., Banerjee A., Quesne C., Tkachuck V.M., "Deformed shape invariance and exactly solvable Hamiltonians with position dependent mass", J. Phys. A 38, no. 13, 2929-2945 (2005).

[10] Gonui B., Kocak M., "Explicit solutions for N-dimensional Schrödinger equations with position dependent massl", J. Math. Phys. 47, 102101 (2006).

[11] Schmidt A., "Wave packet revival for the Schrödinger equation with position dependent massl", Phys. Lett. A 353, 459-462 (2006).

[12] Ganguly A., Kuru S., Negro J., Nieto L.M., "A study of the bound states for square potential wells with position dependent mass", Phys. Lett. A 360, 228-233 (2006).

[13] Ganguly A., Ioffe M.V., Nieto L.M., "A new effective mass Hamiltonian and associated Lam equation: bound states", J. Phys. A 39, no. 47, 14659-14680 (2006). 
[14] Cruz y Cruz S., Negro J., Nieto L.M., "Classical and quantum position-dependent mass harmonic oscillators", Phys. Lett. A 369, no. 5-6, 400-406 (2007).

[15] Ganguly A., Nieto L.M., "Shape-invariant quantum Hamiltonian with position-dependent effective mass through second-order supersymmetry", J. Phys. A 40, 7265-7281 (2007).

[16] Mustafa O., Mazharimousavi S.H., "Ordering Ambiguity Revisited via Position Dependent Mass PseudoMomentum Operators", Int. J. Theor. Phys. 46, no. 7, 1786-1796 (2007).

[17] Cruz y Cruz S., Rosas-Ortiz O., "Position-dependent mass oscillators and coherent states", J. Phys. A: Math. Theor. 42, 185205 (2009).

[18] Mustafa O., Mazharimousavi S.H., "A singular position dependent mass particle in an infite potential welll", Phys. Lett. A 373, no. 3, 325-327 (2009).

[19] Lévai G., Özer O., "An exactly solvable Schrödinger equation with finite positive position-dependent effective mass", J. Math. Phys. 51, 092103 (2010).

[20] Yesiltas O., "The quantum effective mass Hamilton-Jacobi problem”, J. Phys. A: Math. Theor. 43, 095305 (2010).

[21] Lima J.R., Vieira M., Furtado C., Moraes F., Filgueiras C., "Yet another position-dependent mass quantum model", J. Math. Phys. 53, 072101 (2012).

[22] Amir N., Iqbal S., "Algebraic solutions of shape-invariant position-dependent effective mass systems", J. Math. Phys. 57, no. 6, 062105 (2016).

[23] Ioffe M.V., Kolevatova E.V., Nishnianidzeb D.N., "SUSY method for the three-dimensional Schrödinger equation with effective mass", Phys. Lett. A 380, no. 41, 3349.3354 (2016).

[24] Rego-Monteiro M.A., Rodrigues L.M., Curado E.M., "Position-dependent mass quantum Hamiltonians: general approach and duality", J. Phys. A: Math. Theor. 49, no. 12, 125203 (2016).

[25] Lévy-Leblond J.M., "Position-dependent effective mass and Galilean invariance", Phys. Rev. A 52, 1845-1849 (1995).

[26] Chetouani L., Dekar L., Hammann T.F., "Green s functions via path integrals for systems with positiondependent masses", Phys. Rev. A 52, 82-91 (1995).

[27] Dekar L., Chetouani L., Hammann T.F., "An exactly soluble Schrödinger equation with smooth positiondependent mass", J. Math. Phys. 39, 2551-2563 (1998).

[28] Plastino A.R., Rigo A., Casas M., Gracias F., Plastino A., "Supersymmetric approach to quantum systems with position-dependent effective mass", Phys. Rev. A 60, 4318-4325 (1999).

[29] Alhaidari A.D., "Solutions of the nonrelativistic wave equation with position-dependent effective mass", Phys. Rev. A 66, 042116 (2002).

[30] Roy B., Roy P., "PT-symmetric effective mass Schrödinger equations", J. Phys. A 38, no. 50, 11019-11025 (2005).

[31] Quesne C., "First-order intertwining operators and position-dependent mass Schrödinger equations in d dimensions", Ann. Phys. 321, no. 5, 1221-1239 (2006).

[32] Dong S.H., Peña J.J., Pacheco C., García-Arevalo J., "Algebraic approach to the position dependent mass Schrödinger equation for a singular oscillator", Modern Phys. Lett. A 22, no. 14, 1039-1045 (2007).

[33] Quesne C., "Spectrum generating algebras for position-dependent mass oscillator Schrödinger equations", J. Phys. A: Math. Theor. 40, no. 43, 13107-13119 (2007).

[34] Suzkoa A.A., Schulze-Halberg A., "Intertwining operator method and supersymmetry for effective mass Schrödinger equations", Phys. Lett. A 372, no. 37, 5865-5871 (2008).

[35] Kerimov G.A., "The quantum effective mass Hamilton-Jacobi problem", J. Phys. A: Math. Theor. 43, no. 44, 445210 (2009). 
[36] Quesne C., "Point canonical transformation versus deformed shape invariance for position-dependent mass Schrödinger equations", SIGMA Symmetry Integrability Geom. Methods Appl. 5, 046 (2009).

[37] Chithiika Ruby V., Senthilvelan M., "On the construction of coherent states of position dependent mass Schrödinger equation endowed with effective potential", J. Math. Phys. 51, 052106 (2010).

[38] Amir N., Iqbal S., "Ladder operators and associated algebra for position-dependent effective mass systems", European Phys. Lett. 111, 20005 (2015).

[39] Cariñena J.F., Rañada M.F., Santander M., "One-dimensional model of a quantum nonlinear harmonic oscillator", Rep. Math. Phys. 54, no. 2, 285-293 (2004).

[40] Cariñena J.F., Rañada M.F., Santander M., "A quantum exactly solvable nonlinear oscillator with quasiharmonic behaviour", Ann. Phys. 322, no. 2, 434-459 (2007).

[41] Groenewold H.J., "On the principles of elementary quantum mechanics", Physica 12, 405-460 (1946)

[42] Cariñena J.F., Falceto F., Rañada M.F., "A geometric approach to a generalized virial theorem", J. Phys. A: Math. Theor. 45, 395210 (2012).

[43] Cariñena J.F., Gheorghiu I., Martínez E., and Santos P., "Conformal Killing vector fields and a virial theorem", J. Phys. A: Math. Theor. 47, 465206 (2014).

[44] Mathews P.M., Lakshmanan M., "On a unique nonlinear oscillator", Quart. Appl. Math. 32, 215-218 (1974).

[45] Lakshmanan M., Rajasekar S., "Nonlinear dynamics. Integrability, Chaos and Patterns", Advanced Texts in Physics (Springer-Verlag, Berlin, 2003).

[46] Cariñena J.F., Rañada M.F., Santander M., Senthilvelan M., "A non-linear oscillator with quasi-harmonic behaviour: two- and $n$-dimensional oscillators", Nonlinearity 17, no. 5, 1941-1963 (2004).

[47] Chandrasekar V.K., Senthilvelan M., Lakshmanan M., "Unusual Lienard-type nonlinear oscillator", Phys. Rev. E 72 (6), Art. no. 066203 Part 2 (2005).

[48] Midya B., Roy B., "A generalized quantum nonlinear oscillator", J. Phys. A: Math. Theor. 42, no. 28, 285301 (2009).

[49] Bhuvaneswari A., Chandrasekar V.K., Senthilvelan M., Lakshmanan M., "On the complete integrability of a nonlinear oscillator from group theoretical perspective", J. Math. Phys. 53, no. 7, 073504 (2012).

[50] Lakshmanan M., Chandrasekar V.K., "Generating finite dimensional integrable nonlinear dynamical systems", Eur. Phys. J. Special Topics 222, no. 3-4, 665-688 (2013).

[51] Schulze-Halberg A., Morris J.R., "An exactly solvable three-dimensional nonlinear quantum oscillator", J. Math. Phys. 54, no. 11, 112107 (2013).

[52] Rañada M.F., "A quantum nonlinear quasi-harmonic oscillator with an isotonic term", J. Math. Phys. 55, no. 8, 082108 (2014).

[53] Schulze-Halberg A., "Closed-form solutions and supersymmetric partners of the inverted Mathews- Lakshmanan oscillator", European Physical Journal Plus 130, no. 7, Article Number 134 (2015).

[54] Quesne C., "Generalized nonlinear oscillators with quasi-harmonic behaviour: classical solutions", J. Math. Phys. 56, no. 1, 012903 (2015).

[55] Amir N., Iqbal S., "Coherent states for nonlinear harmonic oscillator and some of its properties", J. Math. Phys. 56, no. 6, 062108 (2015).

[56] Bagchi B., Ghosh S., Pal P., Poria S., "Qualitative analysis of certain generalized classes of quadratic oscillator systems", J. Math. Phys. 57, no. 2, 022701 (2016).

[57] Schulze-Halberg A., Roy B., "Generalized quantum nonlinear oscillators: exact solutions and rational extensions", J. Math. Phys. 57, no. 10, 102103 (2016).

[58] Morris J.R., "New scenarios for classical and quantum mechanical systems with position-dependent mass", Quantum Stud. Math. Found. 2, no. 4, 359-370 (2015). 
[59] B. Podolsky, "Quantum mechanically correct form of Hamiltonian function for conservative systems", Phys. Rev. 32, 812-816 (1928).

[60] Schrödinger E., "A method of determining quantum-mechanical eigenvalues and eigenfunctions", Proc. Roy. Irish. Soc. A. 46. 9-16 (1940).

[61] Stevenson A.F.. "Note on the 'Kepler problem' in a spherical space, and the factorization method of solving eigenvalue problems", Phys. Rev. 59, 842-843 (1941).

[62] Higgs P.W., "Dynamical symmetries in a spherical geometry. I.", J. Phys. A. 12, no 3. 309-323 (1979).

[63] Infeld L., Schild A.. "A note on the Kepler problem in a space of constant negative curvature", Phys. Rev. 67, no 3/4. 121-122 (1945).

[64] Bogush AA., Kurochkin Y.A., Otchik V.S. "The quantum-mechanical Kepler problem in three-dimensional Lobachevsky space", Doklady Akad. Nauk BSSR. 24, no 1. 19-22 (1980).

[65] Kurochkin Y., Rybak I., Shoukavy D., "Coherent states on horospheric three-dimensional Lobachevsky space", J. Math. Phys. 57, no. 8, 082111 (2016).

[66] Rozko E.E., Gobcewicz E., "Quantization of systems with internal degrees of freedom in two-dimensional manifolds",Rep. Math. Phys. 73, no. 3, 325-343 (2014),

[67] da Silva L.C.B, Bastos C.C., Ribeiro F.G., "Quantum mechanics of a constrained particle and the problem of prescribed geometry-induced potential", Ann. Phys. 379, 13-33, (2017).

[68] DeWitt B.S., "A Dynamical theory in Curved Spaces I", Rev. Modern Phys. 29, 377-397 (1957).

[69] da Costa R.C.T., "Quantum mechanics of a constrained particle", Phys. Rev. A 23, 1982-1987 (1981).

[70] da Costa R.C.T., "Constraints in quantum mechanics", Phys. Rev. A 25, 2893-2900 (1982).

[71] Encinosa M., Etemadi B., "Energy shifts resulting from surface curvature of quantum nanostructures", Phys. Rev. A 58, 77-81 (1998). 